\title{
An Isotropic Magnetic-Field Transducer Based on the Giant Magnetic Impedance Effect
}

\author{
S. O. Volchkov, E. I. Dukhan, and G. V. Kurlyandskaya \\ Yeltsin Ural Federal University, Yekaterinburg, 400062 Russia \\ e-mail:volchkov@usu.ru,eduhan@pm.convex.ru,galina.kurlyandskaya@usu.ru \\ Received December 20, 2012
}

\begin{abstract}
A comparative analysis of the giant magnetoimpedance effect and the results of a mathematic simulation are presented. This simulation was used for optimizing the topology of a wide-angle magnetic transducer equipped with a sensitive element that consists of two crossed $\mathrm{Fe}_{3} \mathrm{Co}_{67} \mathrm{Cr}_{3} \mathrm{Si}_{15} \mathrm{~B}_{12}$ amorphous ribbons that form different angles between them.
\end{abstract}

Keywords: magnetic impedance, amorphous ribbons, dynamic magnetic permeability

DOI: $10.1134 / \mathrm{S} 1061830913080081$

\section{INTRODUCTION}

One of the important directions of the study of the magnetotransport properties of soft magnetic materials is giant magnetic impedance (GMI), which is the change in the complex resistance (impedance) of a conductor in an applied magnetic field. Owing to the high relative change of impedance (to 500\%/Oe) in relatively moderate magnetic fields and, therefore, to a high sensitivity to the external magnetic field, magnetic transducers (MT) based on GMI elements are used in different engineering applications, such as systems for magnetic nondestructive testing, biodetection, robot techniques, advance testing, and preventing the failure of articles [1-5].

Giant magnetic impedance is a classical electrodynamic effect that is related to a change of the skin layer, namely, to a decrease in the cross section of a ferromagnetic conductor when a high-frequency current flows through it. The magnitude of the skin-layer depth in a nonmagnetic conductor depends only on the ac frequency and the conductivity of the specimen [6]. A ferromagnetic material is characterized by a substantial additional parameter, viz., dynamic magnetic permeability. In the case of soft magnetic materials and a rather high dynamic magnetic permeability, the GMI effect occurs at frequencies that are lower by several orders of magnitude than those for nonmagnetic conductors with the equivalent conductivity. This fact allows the circuit technology and construction of magnetic transducers (MTs) to be simplified substantially. In the general case, the magnetic permeability of ferromagnetic materials is a tensor characteristic. It depends on both the orientations of the external quasistatic magnetic field and the high-frequency current direction and the peculiarities of the effective magnetic anisotropy of the ferromagnetic conductor. The magnetoimpedance response is determined by the peculiarities of the circular magnetic field $h$ produced by ac, whose lines are within planes perpendicular to the specimen axis. Thus, the GMI of amorphous ribbons and conductors that have a cylindrical geometry is determined by the transverse magnetic permeability, $\mu_{\perp}$, and peripheral/circular magnetic permeability, $\mu_{\varphi}$, respectively.

When the position of a conductor, viz., its longitudinal axis relative to the applied magnetic field, changes, the magnitude of the GMI effect also changes, i.e., angular dependence of the magnetic impedance (MI) is observed [5, 7-8]. The MI of most of the known GMI sensors created on the basis of amorphous ribbons depends weakly on the angle between the ribbon axis and external magnetic field direction. This is not always suitable for engineering applications. The design of an anisotropic sensor based on the GMI effect is of high practical interest.

The majority of the studies of the angular dependence of the GMI effect in magnetic transducers based on amorphous ribbons were performed for the simplest case of GMI elements in the form of a single ribbon $[7,8]$. At the same time, there have also been attempts to use more complex configurations of MTs. In particular, a magnetic transducer in the form of two crossed CoFeMoSiB amorphous ribbons that form 


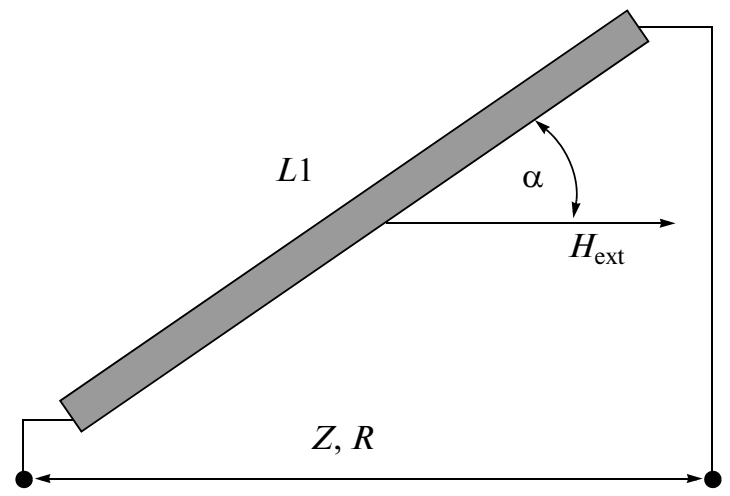

Fig. 1. A magnetic transducer based on a single ribbon $(L 1)$.

an angle of $15^{\circ}$ was studied [9]. It was found that for an angular range of from 0 to $15^{\circ}$ the GMI-effect magnitude for a ribbon-shaped element remains almost unchanged [8-9]. A substantial increase in the angular range of the sensitivity was noted for a magnetic transducer based on a GMI element in the form of two crossed ribbons that form an angle of $15^{\circ}$. However, there is no information about systematic studies of similar configurations of GMI elements, in particular, about mathematical simulation of GMI responses, in the literature.

In the present work, a magnetic-impedance sensitive element that consists of one or two crossed $\mathrm{Fe}_{3} \mathrm{Co}_{67} \mathrm{Cr}_{3} \mathrm{Si}_{15} \mathrm{~B}_{12}$ amorphous ribbons forming different angles was studied while varying the angle of applying an external quasistatic magnetic field.

\section{MATERIALS AND METHODS}

The studies were performed using ribbons of the well-understood $\mathrm{Fe}_{3} \mathrm{Co}_{67} \mathrm{Cr}_{3} \mathrm{Si}_{15} \mathrm{~B}_{12}$ amorphous alloy, which was prepared by melt quenching on a rotary cylinder; this alloy is characterized by a high magnetic permeability, low saturation magnetostriction $\left(\lambda_{S} \sim 10^{-7}\right)$, and high corrosion resistance $[7,10,11]$. No additional heat treatment of the rapidly quenched ribbons was performed. The specimens prepared in such a manner exhibit reproducible characteristics. Measurements of quasistatic magnetic hysteresis loops and studies of the peculiarities of magnetic impedance were performed using specimens in the form of rectangular strips that were $28 \mu \mathrm{m}$ thick, $1 \mathrm{~mm}$ wide, and $50 \mathrm{~mm}$ long. We used sufficiently long specimens to minimize the demagnetizing field along the ribbon axis. All measurements were performed at room temperature $T=23^{\circ} \mathrm{C}$. The effective magnetic anisotropy was found to be longitudinal with a small transverse-component contribution via the surface anisotropy.

We selected specimens of this type using the results of preliminary studies, which showed that the high GMI-effect magnitudes and the sensitivity of the effect to the external magnetic field is reached at relatively low ac frequencies of about $10 \mathrm{MHz}$, namely, the relative change in the impedance $\Delta Z / Z$ and its sensitivity $\Delta(\Delta Z / Z) / \Delta H$ for the specimens without additional treatments reach 160 and $40 \% /$ Oe at relatively low frequencies, respectively $[7,10,11]$.

\section{EXPERIMENTAL STUDIES OF THE GMI OF A MAGNETIC TRANSDUCER BASED ON A SINGLE RIBBON}

At the first stage, we studied the peculiarities of the magnetic-impedance effect of a GMI element in the form of a single ribbon $(L 1)$ while varying the angle $\alpha$ of applying the magnetic field $H_{\text {ext }}($ Fig. 1).

Changes of the magnetic impedance, $Z$, and its ohmic, $R$, and reactance, $X$, components in magnetic fields differing in strength were performed using an Agilent HP E4991A impedance analyzer and a standard measuring scheme $[5,11]$. The high-frequency current in a specimen flowed along its long axis. An external magnetic field $H_{\text {ext }}$ of up to \pm 100 Oe in strength was applied first along the long axis of specimens in alignment with the passed ac and subsequently was varied in a direction depending on the conditions of the experiments. At low absolute values of the magnetic field, the sampling step of magnetic field is $0.08 \mathrm{Oe}$ 


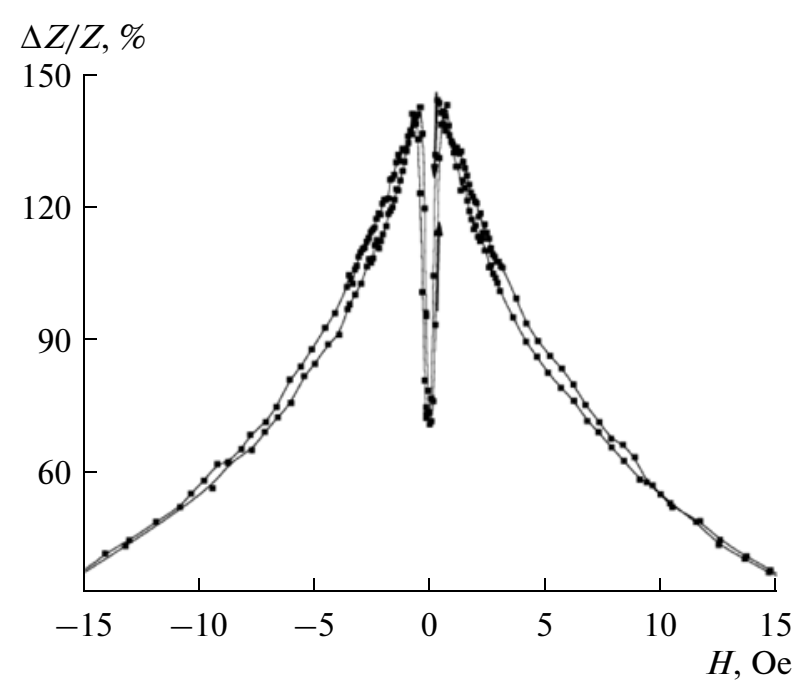

Fig. 2. The relative change of the total impedance $\Delta Z / Z$ for the specimen, $L 1$, as a function of the external magnetic field in a range of \pm 15 Oe.

and exponentially increases as the field strength increases. The measurements were performed first for the decreasing ("descending branch") and then for the increasing ("ascending branch") magnetic field.

The relative changes in the total impedance $\Delta Z / Z$, ohmic resistance $\Delta R / R$, and reactance $\Delta X / X$ were calculated as follows:

$$
\begin{aligned}
& \Delta Z / Z\left(H_{\mathrm{ext}}\right)=\left|Z\left(H_{\mathrm{ext}}\right)-Z\left(H_{\mathrm{max}}\right)\right| / Z\left(H_{\max }\right) ; \\
& \Delta R / R\left(H_{\mathrm{ext}}\right)=\left|R\left(H_{\mathrm{ext}}\right)-R\left(H_{\max }\right)\right| / R\left(H_{\max }\right) ; \\
& \Delta X / X\left(H_{\mathrm{ext}}\right)=\left|X\left(H_{\mathrm{ext}}\right)-X\left(H_{\max }\right)\right| / X\left(H_{\max }\right),
\end{aligned}
$$

where $H_{\max }$ is the magnetic saturation field of a specimen. The amplitude and frequency of the current flowing in the specimen were $10 \mathrm{~mA}$ and $13 \mathrm{MHz}$, respectively; The sensitivities of the total impedance and its ohmic resistance to the external magnetic field were determined as changes in $\Delta Z / Z$ and $\Delta R / R$ magnitudes within the chosen range of the external field $\Delta H$, respectively.

Figure 2 shows the dependence of the relative change in the impedance $\Delta Z / Z$ of specimen $L 1$ on an external magnetic field applied along the long side of a specimen (ribbon axis) $(\alpha=0)$, i.e., in alignment with the high-frequency ac flowing in the specimen. The curve is characterized by a large relative change of the impedance (to 54\%) and high sensitivity to the external magnetic field in the range of relatively small strengths, $H=0.2-0.7$ Oe.

The general character of the GMI curve agrees with the peculiarities of the quasistatic magnetic hysteresis loops, which indicate the existence and prevalence of effective longitudinal magnetic anisotropy with an easy magnetization axis (EMA) in parallel to the long side of the ribbon. The decrease in the impedance in the range of low fields and the occurrence of a double-peak structure indicate the existence of an additional contribution to the effective magnetic anisotropy, which is likely to be related to the presence of a contribution from the transverse magnetic anisotropy component in the surface layer to $2 \mu \mathrm{m}$ thick [13].

As the angle $\alpha$ increases in the range to $70^{\circ}$, a slight shift of the maximum value of the ohmic resistance and impedance in the scale of the external magnetic field from 0.68 to $0.72 \mathrm{Oe}$, a shift of the working point, $H_{\text {w.p. }}$, to the high field range, and a decrease in the sensitivity of both the ohmic resistance $\left(S_{R}\right)$ and impedance $\left(S_{Z}\right)$ (see table) are observed. In the angular range of 70-90 , abrupt changes of all the parameters take place; in this case, the sensitivity of the ohmic resistance $\left(S_{R}\right)$ and total impedance $\left(S_{Z}\right)$ decreases to 0.34 and $0.35 \Omega / \mathrm{Oe}$, respectively.

Figure 3 shows the dependence of the sensitivity of the impedance, $S_{Z}(\Omega / \mathrm{Oe})$, of the GMI element in the form of a single ribbon $L 1$ at the working point (the middle of the range chosen for determining the sensitivity) on the angle $\alpha$ for applying the external magnetic field. It is seen that the sensitivity magnitude is almost unchanged in the range of the angle of applying the external magnetic field from 0 to $45^{\circ}$; in an 
The dependence of a field with the maximum impedance magnitudes, $\left(H_{\text {peak }}\right)$, working point, $\left(H_{\text {work }}\right)$, sensitivity of the ohmic resistance, $\left(S_{R}\right)$, and sensitivity of the impedance, $\left(S_{Z}\right)$, at the working point on the angle of applying the external magnetic field, $(\alpha)$

\begin{tabular}{c|c|c|c|c}
\hline$\alpha$, deg & $H_{\text {peak }}$, Oe & $H_{\text {work }}$ & $S_{R}$ & $S_{Z}$ \\
\hline 0 & 0.68 & 0.35 & 8.02 & 9.87 \\
5 & 0.60 & 0.35 & 8.74 & 9.33 \\
10 & 0.70 & 0.35 & 8.51 & 8.54 \\
15 & 0.60 & 0.32 & 7.62 & 10.08 \\
20 & 0.60 & 0.32 & 9.18 & 8.49 \\
25 & 0.70 & 0.38 & 8.38 & 9.00 \\
30 & 0.60 & 0.38 & 8.32 & 8.85 \\
35 & 0.70 & 0.31 & 9.81 & 9.14 \\
40 & 0.60 & 0.23 & 9.82 & 10.2 \\
45 & 0.52 & 0.21 & 9.53 & 9.06 \\
50 & 0.58 & 0.25 & 9.25 & 8.79 \\
55 & 0.59 & 0.21 & 7.83 & 8.02 \\
60 & 0.60 & 0.27 & 7.71 & 5.69 \\
65 & 0.78 & 0.28 & 7.36 & 6.29 \\
70 & 0.72 & 0.19 & 6.24 & 5.19 \\
75 & 0.82 & 0.30 & 4.94 & 3.45 \\
80 & 1.28 & 0.35 & 3.62 & 2.53 \\
85 & 1.44 & 0.56 & 2.49 & 0.35 \\
90 & 9.60 & 3.60 & 0.34 & \\
\hline
\end{tabular}

angle range from 60 to $90^{\circ}$, an abrupt decrease in the sensitivity of the magnetic transducer takes place. Note that the first quarter of the diagram is symmetrical to the II, III, and IV quarters because of the invariance of the position of a single specimen with respect to the applied external magnetic field.

The magnetoimpedance response is determined by the peculiarities of the circular magnetic field, $h$, produced by ac, whose lines are in planes perpendicular to the ribbon axis; therefore, the impedance is determined by the transverse magnetic permeability $\mu_{\perp}$. In the case of amorphous ribbons, $\mu_{\perp}$ is determined by longitudinal magnetization rotation under a magnetic field, which is the superposition of the circular, $h$, and external, $H$, magnetic fields. The decrease in the magnetization fixing with decreasing longitudinal field $H$, which is accompanied by an increase in the transverse magnetic permeability, must also lead to an increase in the effective magnetic permeability $\left(\mu_{\mathrm{eff}}\right)$ that is observed in the angle range from 0 to $45^{\circ}$. As the angle between the external magnetic field direction and ribbon axis increases, the GMI effect decreases.

\section{THE SIMULATION OF AN MT BASED ON TWO CROSSED RIBBONS}

At the second stage of these studies, in order to decrease the cost and time for the organization of laborconsuming measurements, a mathematical simulation of an MT based on two crossed ribbons was performed and subsequent experimental confirmation of the results we obtained was performed. The simulation of an MT was carried out via the calculation of the resistances of two crossed ribbons that form different angles and are connected in parallel, which are under an external magnetic field; the earlier measurements that were performed for a single specimen were taken into account. The simulation procedure consists in the following (Fig. 4). Each of the calculation cycles was performed for a fixed angle of crossing $\beta$ at a step of $\Delta \beta=5^{\circ}$. The displacement field strength, $H_{\text {w.p. }}$, was chosen so that its projection on the axis of the ribbons, $L 1$ and $L 2$, corresponds to the middle of the working range of the GMI curves of specimens and was taken as unchanged in a calculation cycle. The impedance, $Z_{0}$, ohmic resistance, $R_{0}$, and reactance, $X_{0}$, of the ribbons that were connected in parallel were measured in the absence of an external field. 


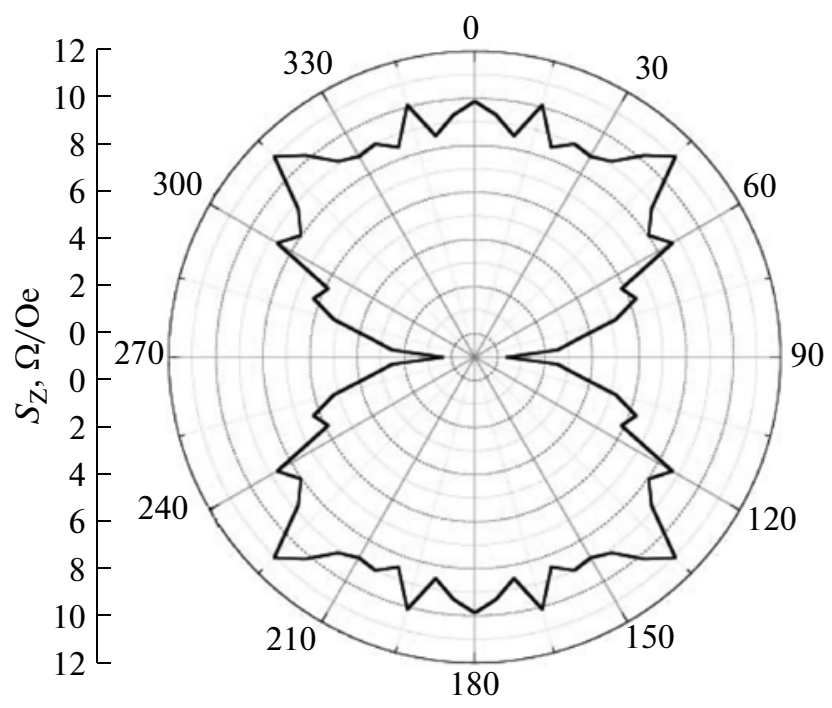

Fig. 3. The dependence of the sensitivity of the impedance $\left(S_{Z}\right)$ of an MT in the form of a single ribbon, $L 1$, on the angle of applying $\alpha$ the external magnetic field.

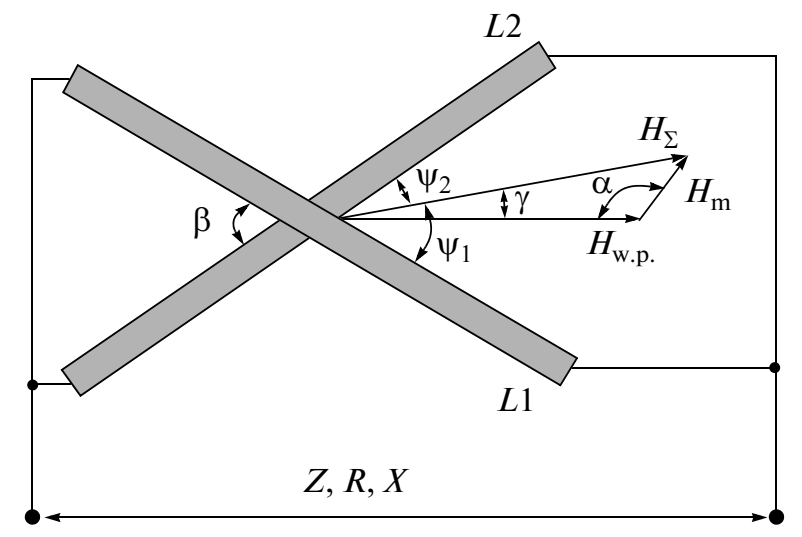

Fig. 4. A simulation of an MT based on two crossed ribbons: $H_{\text {w.p. }}$ is the displacing magnetic field vector (working point); $H_{\mathrm{m}}$ is the (measured) external magnetic field vector; $H_{\Sigma}$ is the resulting magnetic field vector; $\beta$ is the angle between GMI ribbons; $\alpha$ is the angle of applying the external (measuring) magnetic field; $\psi_{1}$ is the angle between the resulting field vector and ribbon $L 1 ; \psi_{2}$ is the angle between the resulting field vector and ribbon $L 2$; and $\gamma$ is the angle forming by the displacing and resulting field vectors.

A low external (measuring) field strength $H_{\mathrm{m}}$ was chosen and, for all calculation cycles, was taken to be half of the working range of the GMI curve that was obtained in the course of the first stage of experiments at an angle of $\delta(\beta)=45^{\circ}$.

The resulting field, $H_{\Sigma}$, and the angles between the $H_{\Sigma}$ vector and specimen axes, $\psi_{1}$ and $\psi_{2}$, were calculated for each angle $\alpha$ of applying the field vector with respect to the bisecting line of the angle between the axes of the specimens at a step of $\Delta \alpha=5^{\circ}$. The ohmic resistance and reactance of both ribbons were determined from tables for the closest magnitudes $H_{\Sigma}, \psi_{1}$, and $\psi_{2}$, and the total impedance for both ribbons, the ohmic resistance, $R_{H}$, and reactance, $X_{H}$, of the MT in the presence of a magnetic field were calculated.

The sensitivity of the MT normalized to the external field for each value of the $\alpha$ and $\beta$ angles was calculated by the expressions:

$$
S_{R}(\alpha, \beta)=\frac{d R}{R_{0}}=\frac{R_{0}-R_{H}}{Z_{0}}
$$




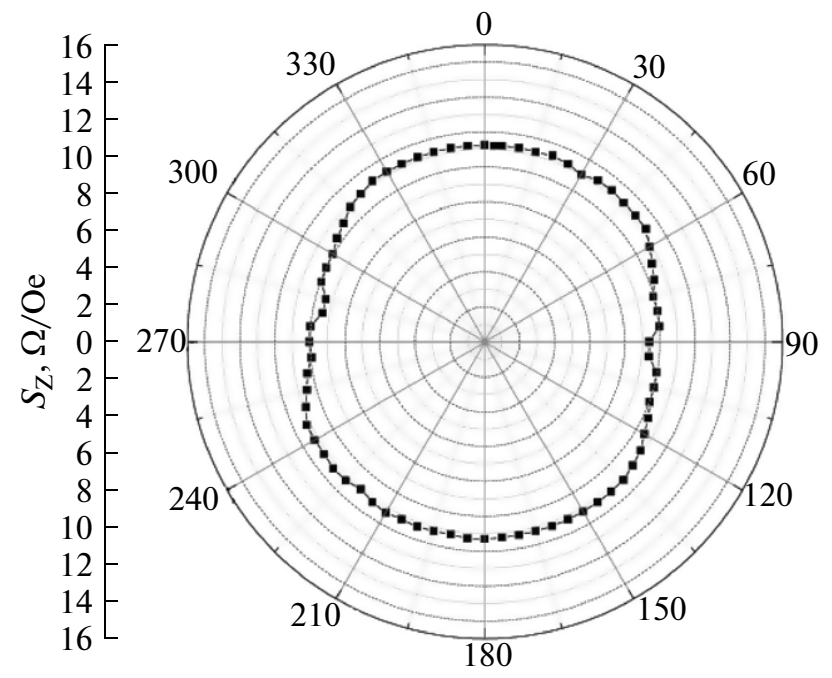

Fig. 5. A simulation of the impedance sensitivity, $S_{Z}$, on the orientation of the system.

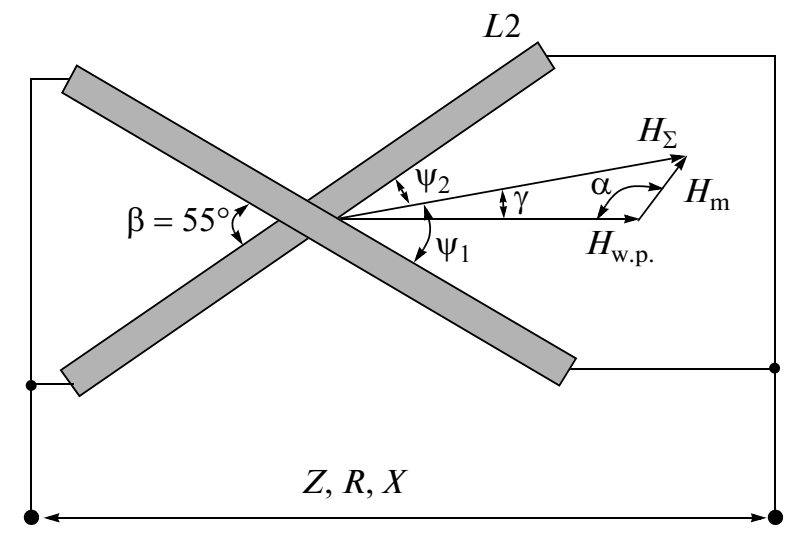

Fig. 6. An experimental study of an MT ( $L 2)$ based on two crossed ribbons $\left(\beta=55^{\circ}\right.$.

$$
\begin{aligned}
& S_{X}(\alpha, \beta)=\frac{\Delta X}{X_{0}}=\frac{X_{0}-X_{H}}{X_{0}} ; \\
& S_{Z}(\alpha, \beta)=\frac{\Delta Z}{Z_{0}}=\frac{Z_{0}-Z_{H}}{Z_{0}} .
\end{aligned}
$$

To perform the calculation in an automated mode, special software was developed. The dependences of the sensitivity $S(\alpha, \beta=$ const) were plotted for each fixed angle $\beta$ between the GIM impedance ribbons using calculated data and the polar coordinate system. The calculations showed that an MT consisting of two crossed ribbons that form an angle $\beta=55^{\circ}$ must yield the most uniform circular characteristic of the sensitivity (Fig. 5). For the angle $\beta=15^{\circ}$, this characteristic is similar to the analogous dependence for an MT consisting of a single ribbon.

\section{EXPERIMENTAL STUDY OF MT BASED ON TWO CROSSED RIBBONS}

At the third stage of these studies, an experimental investigation of the peculiarities of the magnetic impedance effect of a GMI element in the form of two ribbons crossed at an angle of $\beta$, whose optimum magnitude was determined using the results of mathematic simulation and the criterion of uniformity of the circular characteristic of sensitivity, was performed. The impedance, $Z$, and its ohmic resistance, $R$, and reactance, $X$, components in magnetic fields that differed in strength were measured by analogy with 


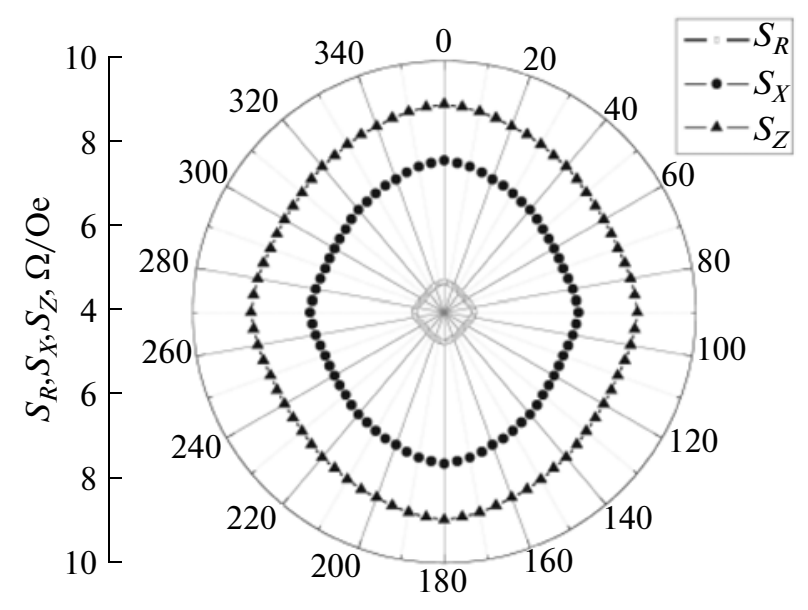

Fig. 7. The dependence of the sensitivity of the ohmic resistance, $\left(S_{R}\right)$, reactance, $\left(S_{X}\right)$, and impedance, $\left(S_{Z}\right)$, of a specimen at the working point (the mid point of the range that was chosen for determining the sensitivity) for an MT based on two ribbons on the angle of applying the external magnetic field in the system.

the measurements that were reported in section II. The GMI element (L2) consisted of two amorphous ribbons crossed at an angle of $\beta=55^{\circ}$ and connected in parallel (Fig. 6).

Initially, the magnetic field was applied along the $H_{\text {w.p. }}$ vector; subsequently, it direction was varied by a certain angle depending on the experimental conditions.

Figure 7 shows the circle diagram for the sensitivity of the ohmic resistance, reactance, and impedance of the magnetic transducer, $L 2$, at the working point. It is seen that the sensitivities of the ohmic resistance, reactance, and impedance were almost unchanged over the entire range of the applied field angles. Thus, a magnetic transducer that was close to isotropic was obtained.

\section{CONCLUSIONS}

A comparative study of the peculiarities of the magnetic properties and giant magnetic impedance of $\mathrm{Fe}_{3} \mathrm{Co}_{67} \mathrm{Cr}_{3} \mathrm{Si}_{15} \mathrm{~B}_{12}$ amorphous ribbons was carried out at different angles of applying a dc magnetic field. The optimization of the parameters of a GMI element, which was performed by mathematic simulation and allows the angular characteristic to be widened, is presented. The results of the mathematic simulation were confirmed experimentally. The experiments showed the monotony of the active and reactive resistances and impedance over the entire angular range from 0 to $360^{\circ}$.

\section{ACKNOWLEDGMENTS}

This work was performed in terms of the theme of the Ural Federal University Magnetodynamics of Nano-Structured Media with High Magnetic Permeability (theme no. 215). We thank A.P. Potapova and V.A. Lukshina for their special support.

\section{REFERENCES}

1. Harrison, E.P., Turney, G.L., and Rowe, H., An impedance magnetometer, Nature, 1935, vol. 135 , p. 961.

2. Makhotkin, V.E., Shurukhin, B.P., Lopatin, V.A., Marchukov, P.Yu., and Levin, Yu.K., Magnetic field sensors based on amorphous ribbons, Sensors and Actuators A, 1991, vol. 27, pp. 759-762.

3. Uchiyama, T., Sompob, P., Mohri, K., and Ishikawa, N., Nondestructive evaluation for structuring steel deformation using amorphous wire MI sensor, J. Mag. Soc. Jpn., 1999, vol. 23, nos 4-2, pp. 1465-1468.

4. Volchkov, S.O. and Kurlyandskaya, G.V., Magnetoimpedance sensor for flat ferromagnetic body detection, Sensor Letters, 2009, vol. 7, pp. 1147-1155.

5. Kurlyandskaya, G.V., de Cos, D., and Volchkov, S.O., Magnetosensitive transducers for nondestructive testing operating on the basis of the giant magnetoimpedance effect: A review, Russ. J. Nondestr. Test., 2009, vol. 45, no. 6, pp. 377-398.

6. Landau, L.D. and Lifshitz, E.M. Electrodynamics of Continuous Media, New York: Pergamon, 1975. 
7. Kurlyandskaya, G.V., Barandiaran, J.M., Vazquez, M., Garcia, D., and Dmitrieva, N.V., Influence of geometrical parameters on the giant magnetoimpedance response in amorphous ribbons, J. Magn. Magn. Mater., 2000, vols. 215-216, pp. 740-742.

8. Kurlyandskaya, G.V. and Levit, V.I., Magnetic Dynabeads ${ }^{\circledR}$ detection by sensitive element based on giant magnetoimpedance, Biosens. Bioelectron, 2005, vol. 20, pp. 1611-1616.

9. Prida, V.M., Garcia-Miquel, Hector, and Kurlyandskaya, G.V., Wide-angle magnetoimpedance fields sensor based on two crossed amorphous ribbons, Sensors and Actuators A, 2008, vol. 142, pp. 496-502.

10. Vázquez, M., Kurlyandskaya, G.V., Muñoz, J.L., Hernando, A., Dmitrieva, N.V., Lukshina, V.A., and Potapov, A.P., Giant magneto-impedance effect in stress-annealed amorphous ribbons, J. De Physique 1998, vol. IV 8, no. 2, pp. Pr2-143-Pr2-146.

11. Volchkov, S.O., Cerdeira, M.A., Gubernatorov, V.V., Duhan, E.I., Potapov, A.P., and Lukshina, V.A., Effects of slight plastic deformation on magnetic properties and giant magnetoimpedance of FeCoCrSiB amorphous ribbons, Chinese Physics Letters, 2007, vol. 24, no. 5, pp. 1357-1360.

12. Saad, A., Garcia, J.A., Kurlyandskaya, G., Santos, J.D., and Elbaile, L., Influence of residual stresses and their relaxation on giant magnetoimpedance of CoFeSiB metallic glasses, Jpn. J. Appl. Phys., 2005, vol. 44, no. 7A, pp. 4939-4944.

Translated by N. Kolchugina 